\title{
q-ANALOGUES OF THE (G.2) SUPERCONGRUENCE OF VAN HAMME
}

\author{
YUDONG LIU AND XIAOXIA WANG*
}

\begin{abstract}
Motivated by the recent research of congruences and $q$-congruences, we provide two different $q$-analogues of the (G.2) supercongruence of Van Hamme through the 'creative microscoping' method, which was devised by Guo and Zudilin. It is a remarkable fact that this is the first time to give direct $q$-analogues of (G.2). In addition, we propose a conjecture related to Swisher's Dwork-type supercongruence (G.3).
\end{abstract}

\section{INTRODUCTION}

In Ramanujan's first letter to Hardy in 1913, he announced that (cf. [1, p. 25, Equation (2)])

$$
\sum_{k=0}^{\infty}(8 k+1) \frac{\left(\frac{1}{4}\right)_{k}^{4}}{k !^{4}}=\frac{2 \sqrt{2}}{\sqrt{\pi} \Gamma\left(\frac{3}{4}\right)^{2}},
$$

along with some similar hypergeometric identities, but he did not give any proofs. Here $(a)_{n}=a(a+1) \cdots(a+n-1)$ denotes the Pochhammer symbol and $\Gamma(x)$ is the Gamma function. The identity (1.1) was ultimately proved by Hardy in [15, p. 495]. In 1997, Van Hamme 22] proposed 13 mysterious $p$-adic analogues of Ramanujan-type $\pi$-formulas, such as,

$$
\sum_{k=0}^{(p-1) / 4}(8 k+1) \frac{\left(\frac{1}{4}\right)_{k}^{4}}{k !^{4}} \equiv p \frac{\Gamma_{p}\left(\frac{1}{2}\right) \Gamma_{p}\left(\frac{1}{4}\right)}{\Gamma_{p}\left(\frac{3}{4}\right)} \quad\left(\bmod p^{3}\right) \quad p \equiv 1 \quad(\bmod 4) .
$$

Here and throughout this paper, $p$ is an odd prime and $\Gamma_{p}(x)$ is the $p$-adic Gamma function [18]. Van Hamme 22] himself proved (C.2), (H.2) and (I.2). Later, Swisher [20] proved that the supercongruence (1.2) is true modulo $p^{4}$ for $p \equiv 1(\bmod 4)$.

During the past few years, the Ramanujan-type congruences and supercongruences, which are viewed as the $p$-adic analogues of Ramanujan-type formulas, have caught attention of many authors (see [3-7, 9, 10, 12 14, 17, 23 25]). Among them, Guo [3-5, 7, 10] and Guo and Wang [12] gave $q$-analogues of most of Van Hamme's 13 conjectural supercongruences by using the $q$-WZ method. Guo and Zudilin [13] introduced the 'creative microscoping' method to prove and reprove many $q$-congruences. Wang and Yue [24]

2010 Mathematics Subject Classification. Primary 33D15; Secondary 11A07, 11B65.

Key words and phrases. basic hypergeometric series; supercongruences; $q$-congruences; $q$-analogue; cyclotomic polynomial;

This work is supported by National Natural Science Foundations of China (11661032). 
succeeded in proving a $q$-analogue of Van Hamme's supercongruence (A.2) for any prime $p \equiv 3(\bmod 4) . \quad$ A $q$-analogue of $($ A.2) for primes $p \equiv 1(\bmod 4)$ was then given by Guo [9]. However, no q-analogues of Van Hamme's (G.2) supercongruence have been found so far.

Recently, Guo and Schlosser [11, Theorems 2] proved that, for even $d \geq 4$ and positive integer $n$ with $n \equiv-1(\bmod d)$,

$$
\sum_{k=0}^{n-1}[2 d k+1] \frac{\left(q ; q^{d}\right)_{k}^{d}}{\left(q^{d} ; q^{d}\right)_{k}^{d}} q^{\frac{d(d-3) k}{2}} \equiv 0 \quad\left(\bmod \Phi_{n}(q)^{2}\right),
$$

which is a $q$-analogue of the $p$-adic analogue of (1.1) for $p \equiv 3(\bmod 4)$ when $d=4$. Moreover, some other interesting $q$-congruences can be found in [16, 19, 21, 26].

In this paper, we shall give two different $q$-analogues of the (G.2) supercongruence of Van Hamme.

Theorem 1. Let $n \equiv 1(\bmod 4)$ be a positive integer. Then

$$
\begin{aligned}
& \sum_{k=0}^{(n-1) / 4}[8 k+1] \frac{\left(q ; q^{4}\right)_{k}^{4}}{\left(q^{4} ; q^{4}\right)_{k}^{4}} q^{2 k} \equiv \frac{\left(q^{2} ; q^{4}\right)_{(n-1) / 4}}{\left(q^{4} ; q^{4}\right)_{(n-1) / 4}}[n] q^{(1-n) / 4} \quad\left(\bmod [n] \Phi_{n}(q)^{2}\right) ; \\
& \sum_{k=0}^{n-1}[8 k+1] \frac{\left(q ; q^{4}\right)_{k}^{4}}{\left(q^{4} ; q^{4}\right)_{k}^{4}} q^{2 k} \equiv \frac{\left(q^{2} ; q^{4}\right)_{(n-1) / 4}}{\left(q^{4} ; q^{4}\right)_{(n-1) / 4}}\left[n q^{(1-n) / 4} \quad\left(\bmod [n] \Phi_{n}(q)^{2}\right) .\right.
\end{aligned}
$$

In fact, setting $n=p \equiv 1(\bmod 4)$ and $q \rightarrow 1$ in (1.4), we get

$$
\sum_{k=0}^{(p-1) / 4}(8 k+1) \frac{\left(\frac{1}{4}\right)_{k}^{4}}{k !^{4}} \equiv \frac{\left(\frac{1}{2}\right)_{(p-1) / 4}}{(1)_{(p-1) / 4}} p \quad\left(\bmod p^{3}\right) .
$$

For prime $p \geq 5$, the $p$-adic Gamma function $\Gamma_{p}$ has the following basic properties [17],

$$
\begin{gathered}
\Gamma_{p}(1)=-1, \quad \Gamma_{p}\left(\frac{1}{2}\right)^{2}=(-1)^{\frac{p+1}{2}}, \quad(a)_{n}=(-1)^{n} \frac{\Gamma_{p}(a+n)}{\Gamma_{p}(a)}, \\
\Gamma_{p}(a+b p) \equiv \Gamma_{p}(a)\left(1+G_{1}(a) b p\right) \quad\left(\bmod p^{2}\right), \quad G_{1}(a)=G_{1}(1-a),
\end{gathered}
$$

where $G_{1}(a):=\Gamma_{p}^{\prime}(a) / \Gamma_{p}(a)$. Then we can rewrite the right-hand side of (1.6) as

$$
\begin{aligned}
\frac{\left(\frac{1}{2}\right)_{(p-1) / 4}}{(1)_{(p-1) / 4}} p=\frac{\Gamma_{p}(1) \Gamma_{p}\left(\frac{1}{4}+\frac{p}{4}\right)}{\Gamma_{p}\left(\frac{1}{2}\right) \Gamma_{p}\left(\frac{3}{4}+\frac{p}{4}\right)} p & \equiv-\frac{\Gamma_{p}\left(\frac{1}{4}\right)\left(1+G_{1}\left(\frac{1}{4}\right) \frac{p}{4}\right)}{\Gamma_{p}\left(\frac{1}{2}\right) \Gamma_{p}\left(\frac{3}{4}\right)\left(1+G_{1}\left(\frac{3}{4}\right) \frac{p}{4}\right)} p \quad\left(\bmod p^{3}\right) \\
& =\frac{\Gamma_{p}\left(\frac{1}{2}\right) \Gamma_{p}\left(\frac{1}{4}\right)}{\Gamma_{p}\left(\frac{3}{4}\right)} p \quad\left(\bmod p^{3}\right)
\end{aligned}
$$

which is just the right-hand side of Van Hamme's (G.2) supercongruence.

Likewise, we have the following supercongruence as $q \rightarrow 1$ in (1.5):

$$
\sum_{k=0}^{p-1}(8 k+1) \frac{\left(\frac{1}{4}\right)_{k}^{4}}{k !^{4}} \equiv p \frac{\Gamma_{p}\left(\frac{1}{2}\right) \Gamma_{p}\left(\frac{1}{4}\right)}{\Gamma_{p}\left(\frac{3}{4}\right)} \quad\left(\bmod p^{3}\right) \quad p \equiv 1 \quad(\bmod 4),
$$


which is an equivalent form of (1.2), since $\left(\frac{1}{4}\right)_{k} / k ! \equiv 0(\bmod p)$ for $(p-1) / 4<k \leq p-1$.

Theorem 2. Let $n \equiv 1(\bmod 4)$ be a positive integer. Then, modulo $[n]_{q^{2}} \Phi_{n}\left(q^{2}\right)^{2}$,

$$
\begin{aligned}
\sum_{k=0}^{(n-1) / 4}[8 k+1]_{q^{2}}[8 k+1]^{2} \frac{\left(q^{2} ; q^{8}\right)_{k}^{4}}{\left(q^{8} ; q^{8}\right)_{k}^{4}} q^{-4 k} & \equiv-\frac{2[n]_{q^{2}}\left(q^{4} ; q^{8}\right)_{(n-1) / 4}}{\left(1+q^{2}\right)\left(q^{8} ; q^{8}\right)_{(n-1) / 4}} q^{(3-n) / 2}, \\
\sum_{k=0}^{n-1}[8 k+1]_{q^{2}}[8 k+1]^{2} \frac{\left(q^{2} ; q^{8}\right)_{k}^{4}}{\left(q^{8} ; q^{8}\right)_{k}^{4}} q^{-4 k} & \equiv-\frac{2[n]_{q^{2}}\left(q^{4} ; q^{8}\right)_{(n-1) / 4}}{\left(1+q^{2}\right)\left(q^{8} ; q^{8}\right)_{(n-1) / 4}} q^{(3-n) / 2} .
\end{aligned}
$$

Leting $n=p$ and $q \rightarrow-1$ in (1.7), we obtain (G.2) once more. Further, we have the following similar supercongruences by taking $q \rightarrow 1$ in Theorem 2 :

$$
\begin{array}{cc}
\sum_{k=0}^{(p-1) / 4}(8 k+1)^{3} \frac{\left(\frac{1}{4}\right)_{k}^{4}}{k !^{4}} \equiv-\frac{\Gamma_{p}\left(\frac{1}{2}\right) \Gamma_{p}\left(\frac{1}{4}\right)}{\Gamma_{p}\left(\frac{3}{4}\right)} p & \left(\bmod p^{3}\right), \\
\sum_{k=0}^{p-1}(8 k+1)^{3} \frac{\left(\frac{1}{4}\right)_{k}^{4}}{k !^{4}} \equiv-\frac{\Gamma_{p}\left(\frac{1}{2}\right) \Gamma_{p}\left(\frac{1}{4}\right)}{\Gamma_{p}\left(\frac{3}{4}\right)} p & \left(\bmod p^{3}\right) .
\end{array}
$$

As for prerequisites, the reader is expected to know the standard $q$-notation. For an indeterminate $q,(a ; q)_{n}=(1-a)(1-a q) \cdots\left(1-a q^{n-1}\right)$ is called the $q$-shifted factorial. For convenience, we compactly write $\left(a_{1}, a_{2}, \ldots, a_{m} ; q\right)_{n}=\left(a_{1} ; q\right)_{n}\left(a_{2} ; q\right)_{n} \cdots\left(a_{m} ; q\right)_{n}$ for the product of $q$-shifted factorials. Moreover, $\Phi_{n}(q)$ denotes the $n$-th cyclotomic polynomial in $q$, which is defined as

$$
\Phi_{n}(q)=\prod_{\substack{1 \leqslant k \leqslant n \\ \operatorname{gcd}(n, k)=1}}\left(q-\zeta^{k}\right)
$$

where $\zeta$ is an $n$-th primitive root of unity. Furthermore, for arbitrary integer $n,[n]=$ $[n]_{q}=\left(1-q^{n}\right) /(1-q)$ is the $q$-integer.

The rest of the paper is organized as follows. We shall prove Theorems 1 and 2 based on Rogers' nonterminating ${ }_{6} \phi_{5}$ summation and Watson's ${ }_{8} \phi_{7}$ transformation in the Sections 2 and 3. Certain generalizations of Theorems 1 and 2 will be given in Section 4 . Finally, in Section 5, we will propose a $q$-analogue of Swisher's Dwork-type conjecture supercongruence (G.3) with $p \equiv 1(\bmod 4)$.

\section{Proof of Theorem 1}

We start with Rogers' nonterminating ${ }_{6} \phi_{5}$ summation (cf. [2, Appendix (II.20)]):

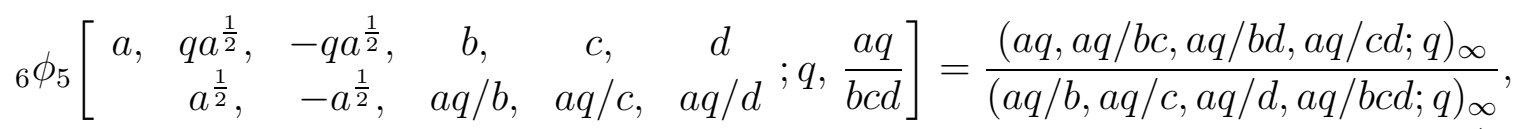

where $|a q / b c d|<1$ for convergence.

Also, the following lemmas are needed in our proof. 
Lemma 1. Let $d \geq 2, m>1,0 \leq s \leq m-1, t$ be integers with $\operatorname{gcd}(d, t)=1$ and $d s \equiv-t$ $(\bmod m)$. Then, for $0 \leq k \leq s$, we have

$$
\frac{\left(a q^{t} ; q^{d}\right)_{s-k}}{\left(q^{d} / a ; q^{d}\right)_{s-k}} \equiv(-a)^{s-2 k} q^{s(d s-d+2 t) / 2+(d-t) k} \frac{\left(a q^{t} ; q^{d}\right)_{k}}{\left(q^{d} / a ; q^{d}\right)_{k}} \quad\left(\bmod \Phi_{m}(q)\right) .
$$

Proof. Since $q^{m} \equiv 1\left(\bmod \Phi_{m}(q)\right)$, we have

$$
\begin{aligned}
\frac{\left(a q^{t} ; q^{d}\right)_{s}}{\left(q^{d} / a ; q^{d}\right)_{s}} & =\frac{\left(1-a q^{t}\right)\left(1-a q^{t+d}\right) \cdots\left(1-a q^{t+d s-d}\right)}{\left(1-q^{d} / a\right)\left(1-q^{2 d} / a\right) \cdots\left(1-q^{d s} / a\right)} \\
& \equiv \frac{\left(1-a q^{t}\right)\left(1-a q^{t+d}\right) \cdots\left(1-a q^{t+d s-d}\right)}{\left(1-q^{d-d s-t} / a\right)\left(1-q^{2 d-d s-t} / a\right) \cdots\left(1-q^{-t} / a\right)} \\
& =(-a)^{s} q^{s(2 t+d s-d) / 2} \quad\left(\bmod \Phi_{m}(q)\right) .
\end{aligned}
$$

For $0 \leq k \leq s$, we obtain

$$
\begin{aligned}
\frac{\left(a q^{t} ; q^{d}\right)_{s-k}}{\left(q^{d} / a ; q^{d}\right)_{s-k}} & =\frac{\left(a q^{t} ; q^{d}\right)_{s}}{\left(q^{d} / a ; q^{d}\right)_{s}} \frac{\left(1-q^{d s-(k-1) d} / a\right) \cdots\left(1-q^{d s} / a\right)}{\left(1-a q^{d s-d k+t}\right) \cdots\left(1-a q^{d s-d+t}\right)} \\
& \equiv \frac{\left(a q^{t} ; q^{d}\right)_{s}}{\left(q^{d} / a ; q^{d}\right)_{s}} \frac{\left(1-q^{-d k+d-t} / a\right) \cdots\left(1-q^{-t} / a\right)}{\left(1-a q^{-d k}\right) \cdots\left(1-a q^{-d}\right)} \quad\left(\bmod \Phi_{m}(q)\right) \\
& \equiv(-a)^{s-2 k} q^{s(d s-d+2 t) / 2+(d-t) k} \frac{\left(a q^{t} ; q^{d}\right)_{k}}{\left(q^{d} / a ; q^{d}\right)_{k}} \quad\left(\bmod \Phi_{m}(q)\right)
\end{aligned}
$$

as desired.

Lemma 2. Let $m>1, d \geq 2, t$ be integers with $\operatorname{gcd}(d, m)=1$ and $\operatorname{gcd}(d, t)=1$. Then

$$
\sum_{k=0}^{m-1}[2 d k+t] \frac{\left(q^{t} ; q^{d}\right)_{k}^{2}\left(a q^{t} ; q^{d}\right)_{k}\left(q^{t} / a ; q^{d}\right)_{k}}{\left(q^{d} ; q^{d}\right)_{k}^{2}\left(a q^{d} ; q^{d}\right)_{k}\left(q^{d} / a ; q^{d}\right)_{k}} q^{(d-2 t) k} \equiv 0 \quad\left(\bmod \Phi_{m}(q)\right) .
$$

Proof. Since $\operatorname{gcd}(d, m)=1$, there exists a unique integer $s$ with $0 \leq s \leq m-1$ and $d s \equiv-t(\bmod m)$. Applying Lemma 1 , for $0 \leq k \leq s$, we have

$$
\begin{aligned}
& {[2 d(s-k)+t] \frac{\left(q^{t} ; q^{d}\right)_{s-k}^{2}\left(a q^{t} ; q^{d}\right)_{s-k}\left(q^{t} / a ; q^{d}\right)_{s-k}}{\left(q^{d} ; q^{d}\right)_{s-k}^{2}\left(a q^{d} ; q^{d}\right)_{s-k}\left(q^{d} / a ; q^{d}\right)_{s-k}} q^{(d-2 t)(s-k)}} \\
& \equiv-[2 d k+t] \frac{\left(q^{t} ; q^{d}\right)_{k}^{2}\left(a q^{t} ; q^{d}\right)_{k}\left(q^{t} / a ; q^{d}\right)_{k}}{\left(q^{d} ; q^{d}\right)_{k}^{2}\left(a q^{d} ; q^{d}\right)_{k}\left(q^{d} / a ; q^{d}\right)_{k}} q^{(d-2 t) k} \quad\left(\bmod \Phi_{m}(q)\right) .
\end{aligned}
$$

Hence, if $s$ is odd, then we get

$$
\sum_{k=0}^{s}[2 d k+t] \frac{\left(q^{t} ; q^{d}\right)_{k}^{2}\left(a q^{t} ; q^{d}\right)_{k}\left(q^{t} / a ; q^{d}\right)_{k}}{\left(q^{d} ; q^{d}\right)_{k}^{2}\left(a q^{d} ; q^{d}\right)_{k}\left(q^{d} / a ; q^{d}\right)_{k}} q^{(d-2 t) k} \equiv 0 \quad\left(\bmod \Phi_{m}(q)\right) .
$$

On the other hand, if $s$ is even, then the middle term of (2.4) contains the factor $\left[2 d\left(\frac{s}{2}\right)+\right.$ $t]=[d s+t]$, which is congruent to 0 modulo $\Phi_{m}(q)$. Then we arrive at (2.4) for $0 \leq s \leq$ 
$m-1$. Furthermore, since $\left(q^{t} ; q^{d}\right)_{k} /\left(q^{d} ; q^{d}\right)_{k} \equiv 0\left(\bmod \Phi_{m}(q)\right)$ for $s<k \leq m-1$, we directly obtain (2.3). This completes the proof of the lemma.

We now present the following parametric generalization of Theorem 1 .

Theorem 3. Let $n \equiv 1(\bmod 4)$ be a positive integer. For any indeterminate a, modulo $[n]\left(1-a q^{n}\right)\left(a-q^{n}\right)$, we have

$$
\sum_{k=0}^{(n-1) / 4}[8 k+1] \frac{\left(q ; q^{4}\right)_{k}^{2}\left(a q ; q^{4}\right)_{k}\left(q / a ; q^{4}\right)_{k}}{\left(q^{4} ; q^{4}\right)_{k}^{2}\left(a q^{4} ; q^{4}\right)_{k}\left(q^{4} / a ; q^{4}\right)_{k}} q^{2 k} \equiv \frac{\left(q^{2} ; q^{4}\right)_{(n-1) / 4}}{\left(q^{4} ; q^{4}\right)_{(n-1) / 4}}[n] q^{(1-n) / 4} .
$$

Proof. For $a=q^{n}$ or $a=q^{-n}$, the left-hand side of (2.5) is equal to

$$
\sum_{k=0}^{(n-1) / 4}[8 k+1] \frac{\left(q ; q^{4}\right)_{k}^{2}\left(q^{1+n} ; q^{4}\right)_{k}\left(q^{1-n} ; q^{4}\right)_{k}}{\left(q^{4} ; q^{4}\right)_{k}^{2}\left(q^{4+n} ; q^{4}\right)_{k}\left(q^{4-n} ; q^{4}\right)_{k}} q^{2 k}
$$

which by Rogers' summation (2.1) with the parameter substitutions $q \mapsto q^{4}, a=d=q$, $b=q^{1-n}$ and $c=q^{1+n}$ can be written as

$$
\begin{aligned}
& { }_{6} \phi_{5}\left[\begin{array}{cccccc}
q, & q^{\frac{9}{2}}, & -q^{\frac{9}{2}}, & q^{1-n}, & q^{1+n}, & q \\
& q^{\frac{1}{2}}, & -q^{\frac{1}{2}}, & q^{4+n}, & q^{4-n}, & q^{4}
\end{array} q^{4}, q^{2}\right] \\
& =\frac{\left(q^{5}, q^{3}, q^{3-n}, q^{3+n} ; q^{4}\right)_{\infty}}{\left(q^{4-n}, q^{4+n}, q^{4}, q^{2} ; q^{4}\right)_{\infty}} \\
& =\frac{\left(q^{2} ; q^{4}\right)_{(n-1) / 4}}{\left(q^{4} ; q^{4}\right)_{(n-1) / 4}}[n] q^{(1-n) / 4} .
\end{aligned}
$$

This means that the $q$-congruence (2.5) holds modulo $1-a q^{n}$ and $a-q^{n}$.

In what follows we shall prove

$$
\sum_{k=0}^{(n-1) / 4}[8 k+1] \frac{\left(q ; q^{4}\right)_{k}^{2}\left(a q ; q^{4}\right)_{k}\left(q / a ; q^{4}\right)_{k}}{\left(q^{4} ; q^{4}\right)_{k}^{2}\left(a q^{4} ; q^{4}\right)_{k}\left(q^{4} / a ; q^{4}\right)_{k}} q^{2 k} \equiv 0 \quad(\bmod [n]) .
$$

Let $\zeta \neq 1$ be an $n$-th unity root, not necessarily primitive. Then $\zeta$ must be a primitive $m_{1}$-th root of unity with $m_{1} \mid n$. Since $\operatorname{gcd}\left(m_{1}, 4\right)=1$, there exists a unique integer $s_{1}$ with $0<s_{1} \leq m_{1}-1$ and $4 s_{1} \equiv-1\left(\bmod m_{1}\right)$. Let $c_{q}(k)$ denote the $k$-th term on the left-hand side in (2.5), i.e,

$$
c_{q}(k)=[8 k+1] \frac{\left(q ; q^{4}\right)_{k}^{2}\left(a q ; q^{4}\right)_{k}\left(q / a ; q^{4}\right)_{k}}{\left(q^{4} ; q^{4}\right)_{k}^{2}\left(a q^{4} ; q^{4}\right)_{k}\left(q^{4} / a ; q^{4}\right)_{k}} q^{2 k} .
$$

Letting $d=4, t=1, m=m_{1}$ in (2.3) and combining (2.4), we have

$$
\sum_{k=0}^{m_{1}-1} c_{\zeta}(k)=\sum_{k=0}^{s_{1}} c_{\zeta}(k)=0
$$


For $0 \leq k \leq m_{1}-1$, the following limit holds:

$$
\lim _{q \rightarrow \zeta} \frac{c_{q}\left(l m_{1}+k\right)}{c_{q}\left(l m_{1}\right)}=c_{\zeta}(k)
$$

Then, we obtain

$$
\begin{gathered}
\sum_{k=0}^{\frac{n-1}{4}} c_{\zeta}(k)=\sum_{l=0}^{\frac{n-4 s_{1}-1}{4 m_{1}}-1} c_{\zeta}\left(l m_{1}\right) \sum_{k=0}^{m_{1}-1} c_{\zeta}(k)+c_{\zeta}\left(\left(n-4 s_{1}-1\right) / 4\right) \sum_{k=0}^{s_{1}} c_{\zeta}(k)=0 ; \\
\sum_{k=0}^{n-1} c_{\zeta}(k)=\sum_{l=0}^{n / m_{1}-1} c_{\zeta}\left(l m_{1}\right) \sum_{k=0}^{m_{1}-1} c_{\zeta}(k)=0 .
\end{gathered}
$$

It follows that

$$
\sum_{k=0}^{M}[8 k+1] \frac{\left(q ; q^{4}\right)_{k}^{2}\left(a q ; q^{4}\right)_{k}\left(q / a ; q^{4}\right)_{k}}{\left(q^{4} ; q^{4}\right)_{k}^{2}\left(a q^{4} ; q^{4}\right)_{k}\left(q^{4} / a ; q^{4}\right)_{k}} q^{2 k} \equiv 0 \quad\left(\bmod \Phi_{m_{1}}(q)\right)
$$

where $M=(n-1) / 4$ or $n-1$. Noting that

$$
\prod_{m_{1} \mid n, m_{1}>1} \Phi_{m_{1}}(q)=[n]
$$

we immediately get

$$
\sum_{k=0}^{M}[8 k+1] \frac{\left(q ; q^{4}\right)_{k}^{2}\left(a q ; q^{4}\right)_{k}\left(q / a ; q^{4}\right)_{k}}{\left(q^{4} ; q^{4}\right)_{k}^{2}\left(a q^{4} ; q^{4}\right)_{k}\left(q^{4} / a ; q^{4}\right)_{k}} q^{2 k} \equiv 0 \quad(\bmod [n]) .
$$

Since $[n], a-q^{n}$ and $1-a q^{n}$ are pairwise relatively prime polynomials, we complete the proof of the theorem.

Proof of Theorem 1. For $k$ in the range $0 \leq k \leq(n-1) / 4$, since $\operatorname{gcd}(n, 4)=1$, the numbers $4,8 \cdots 4(n-1)$ are all not divisible by $n$. So that the limit $a \rightarrow 1$ of the denominator related to $a$ in (2.5) is relatively prime to $\Phi_{n}(q)$. On the other hand, the limit $\left(1-a q^{n}\right)\left(a-q^{n}\right)$ as $a \rightarrow 1$ contains the factor $\Phi_{n}(q)^{2}$. Thus, letting $a \rightarrow 1$ in (2.5), we conclude that (1.4) is true modulo $\Phi_{n}(q)^{3}$. Setting $a \rightarrow 1$ in (2.10), we get

$$
\sum_{k=0}^{M}[8 k+1] \frac{\left(q ; q^{4}\right)_{k}^{4}}{\left(q^{4} ; q^{4}\right)_{k}^{4}} q^{2 k} \equiv 0 \quad(\bmod [n]),
$$

which means that (1.4) also holds modulo $[n]$. Since the least common multiple of $[n]$ and $\Phi_{n}(q)^{3}$ is $[n] \Phi_{n}(q)^{2}$, we obtain (1.4). Moreover, in view of $\left(q ; q^{4}\right)_{k}^{4} /\left(q^{4} ; q^{4}\right)_{k}^{4} \equiv 0$ $\left(\bmod \Phi_{n}(q)^{4}\right)$ for $(n-1) / 4<k \leq n-1$, we arrive at (1.5). This completes the proof. 


\section{Proof of Theorem 2}

In this section, we need Watson's ${ }_{8} \phi_{7}$ transformation formula (cf. [2, Appendix (II.17)])

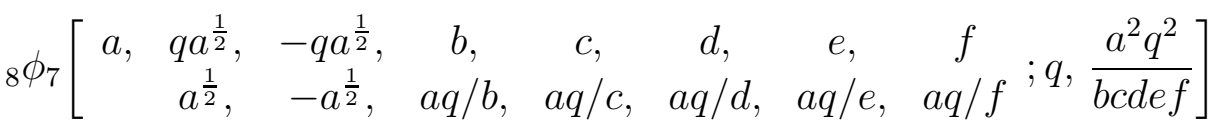

$$
\begin{aligned}
& =\frac{(a q, a q / d e, a q / d f, a q / e f ; q)_{\infty}}{(a q / d, a q / e, a q / f, a q / \operatorname{def} ; q)_{\infty}}{ }_{4} \phi_{3}\left[\begin{array}{c}
a q / b c, d, e, f \\
a q / b, a q / c, \text { def } / a
\end{array} ; q, q\right]
\end{aligned}
$$

to accomplish our proof. Moreover, we require the following lemma.

Lemma 3. Let $m>1, d \geq 2, t$ be integers with $\operatorname{gcd}(d, m)=1$ and $\operatorname{gcd}(d, t)=1$. Then

$$
\sum_{k=0}^{m-1}[2 d k+t]_{q^{2}}[2 d k+t]^{2} \frac{\left(q^{2 t} ; q^{2 d}\right)_{k}^{2}\left(a q^{2 t} ; q^{2 d}\right)_{k}\left(q^{2 t} / a ; q^{2 d}\right)_{k}}{\left(q^{2 d} ; q^{2 d}\right)_{k}^{2}\left(a q^{2 d} ; q^{2 d}\right)_{k}\left(q^{2 d} / a ; q^{2 d}\right)_{k}} q^{-4 t k} \equiv 0 \quad\left(\bmod \Phi_{m}\left(q^{2}\right)\right) .
$$

Proof. Setting $q \mapsto q^{2}$ in (2.2), we get

$$
\frac{\left(a q^{2 t} ; q^{2 d}\right)_{s-k}}{\left(q^{2 d} / a ; q^{2 d}\right)_{s-k}} \equiv(-a)^{s-2 k} q^{s(d s-d+2 t)+2(d-t) k} \frac{\left(a q^{2 t} ; q^{2 d}\right)_{k}}{\left(q^{2 d} / a ; q^{2 d}\right)_{k}} \quad\left(\bmod \Phi_{m}\left(q^{2}\right)\right),
$$

where $0 \leq s \leq m-1$ and $d s \equiv-t(\bmod m)$. Similarly as the proof of Lemma 2, by (3.3), we can see that the sum of the $k$-th and $(s-k)$-th terms on the left-hand side of (3.2) are congruent to zero modulo $\Phi_{m}\left(q^{2}\right)$ when $k \neq s / 2$. So the following $q$-congruence is true when $s$ is odd:

$$
\sum_{k=0}^{s}[2 d k+t]_{q^{2}}[2 d k+t]^{2} \frac{\left(q^{2 t} ; q^{2 d}\right)_{k}^{2}\left(a q^{2 t} ; q^{2 d}\right)_{k}\left(q^{2 t} / a ; q^{2 d}\right)_{k}}{\left(q^{2 d} ; q^{2 d}\right)_{k}^{2}\left(a q^{2 d} ; q^{2 d}\right)_{k}\left(q^{2 d} / a ; q^{2 d}\right)_{k}} q^{-4 t k} \equiv 0 \quad\left(\bmod \Phi_{m}\left(q^{2}\right)\right)
$$

On the other hand, if $s$ is even, then $\left[2 d\left(\frac{s}{2}\right)+t\right]_{q^{2}}=[d s+t]_{q^{2}} \equiv 0\left(\bmod \Phi_{m}\left(q^{2}\right)\right)$. This means that (3.4) holds for any arbitrary integer $0 \leq s \leq m-1$. Since $\left(q^{2 t} ; q^{2 d}\right)_{k} /\left(q^{2 d} ; q^{2 d}\right)_{k} \equiv$ $0\left(\bmod \Phi_{m}\left(q^{2}\right)\right)$ for $s<k \leq m-1$, we immediately arrive at (3.2).

In order to prove Theorem 2, we also need to establish the following parametric generalization.

Theorem 4. Let $n \equiv 1(\bmod 4)$ be a positive integer. Then, for any indeterminate a, modulo $[n]_{q^{2}}\left(1-a q^{2 n}\right)\left(a-q^{2 n}\right)$, we have

$$
\begin{aligned}
& \sum_{k=0}^{(n-1) / 4}[8 k+1]_{q^{2}}[8 k+1]^{2} \frac{\left(q^{2} ; q^{8}\right)_{k}^{2}\left(a q^{2} ; q^{8}\right)_{k}\left(q^{2} / a ; q^{8}\right)_{k}}{\left(q^{8} ; q^{8}\right)_{k}^{2}\left(a q^{8} ; q^{8}\right)_{k}\left(q^{8} / a ; q^{8}\right)_{k}} q^{-4 k} \\
& \equiv[n]_{q^{2}} \frac{\left(q^{4} ; q^{8}\right)_{(n-1) / 4}}{\left(q^{8} ; q^{8}\right)_{(n-1) / 4}} q^{-(n-1) / 2}\left(1-\frac{\left(1-a q^{2}\right)\left(1-q^{2} / a\right)}{(1-q)^{2}\left(1+q^{2}\right)}\right) .
\end{aligned}
$$


Proof. For $a=q^{2 n}$ or $a=q^{-2 n}$, the left-hand side of (3.5) is equal to

$$
\begin{aligned}
& \sum_{k=0}^{(n-1) / 4}[8 k+1]_{q^{2}}[8 k+1]^{2} \frac{\left(q^{2} ; q^{8}\right)_{k}^{2}\left(q^{2+2 n} ; q^{8}\right)_{k}\left(q^{2-2 n} ; q^{8}\right)_{k}}{\left(q^{8} ; q^{8}\right)_{k}^{2}\left(q^{8+2 n} ; q^{8}\right)_{k}\left(q^{8-2 n} ; q^{8}\right)_{k}} q^{-4 k} \\
& ={ }_{8} \phi_{7}\left[\begin{array}{cccccccc}
q^{2}, & q^{9}, & -q^{9}, & q^{9}, & q^{9}, & q^{2}, & q^{2-2 n}, & q^{2+2 n} \\
q, & -q, & q, & q, & q^{8}, & q^{8-2 n}, & q^{8+2 n}
\end{array} q^{8}, q^{-4}\right],
\end{aligned}
$$

where the ${ }_{8} \phi_{7}$ series can be evaluated by Watson's ${ }_{8} \phi_{7}$ transformation (3.1) with the parameter substitutions $q \mapsto q^{8}, a=d=q^{2}, b=c=q^{9}, e=q^{2+2 n}$ and $f=q^{2-2 n}$ as follows:

$$
\begin{aligned}
& \frac{\left(q^{10}, q^{6}, q^{6-2 n}, q^{6+2 n} ; q^{8}\right)_{\infty}}{\left(q^{8}, q^{4}, q^{8-2 n}, q^{8+2 n} ; q^{8}\right)_{\infty}} \phi_{3}\left[\begin{array}{c}
q^{-8}, q^{2}, q^{2+2 n}, q^{2-2 n} ; q^{8}, q^{8} \\
q, q, q^{4}
\end{array}\right. \\
& =[n]_{q^{2}} \frac{\left(q^{4} ; q^{8}\right)_{(n-1) / 4}}{\left(q^{8} ; q^{8}\right)_{(n-1) / 4}} q^{-(n-1) / 2}\left(1-\frac{\left(1-q^{2+2 n}\right)\left(1-q^{2-2 n}\right)}{(1-q)^{2}\left(1+q^{2}\right)}\right) .
\end{aligned}
$$

This means that the $q$-congruence (3.5) modulo $\left(1-a q^{2 n}\right)\left(a-q^{2 n}\right)$ holds true. Moreover, for $n>1$, let $\eta \neq 1$ be an $n$-th unity root, not necessarily primitive. Then $\eta$ must be a primitive $m_{2}$-th root of unity with $m_{2} \mid n$. Owing to $\operatorname{gcd}\left(m_{2}, 4\right)=1$, there exists a unique integer $s_{2}$ with $0<s_{2} \leq m_{2}-1$ and $4 s_{2} \equiv-1\left(\bmod m_{2}\right)$. Setting $d=4, t=1, s=s_{2}$, $m=m_{2}$ in (3.2) and (3.4) we have

$$
\sum_{k=0}^{m_{2}-1} p_{\eta}(k)=\sum_{k=0}^{s_{2}} p_{\eta}(k)=0 \quad \text { and } \quad \sum_{k=0}^{m_{2}-1} p_{-\eta}(k)=\sum_{k=0}^{s_{2}} p_{-\eta}(k)=0,
$$

where $p_{q}(k)$ denotes the $k$-th term on the left-hand side of (3.5). Also, we can calculate that

$$
\lim _{q \rightarrow \eta} \frac{p_{q}\left(l m_{2}+k\right)}{p_{q}\left(l m_{2}\right)}=p_{\eta}(k) .
$$

Likewise, we get the following result

$$
\begin{aligned}
& \sum_{k=0}^{(n-1) / 4} p_{\eta}(k)=\sum_{\ell=0}^{\frac{n-4 s_{2}-1}{4 m_{2}}-1} p_{\eta}\left(\ell m_{2}\right) \sum_{k=0}^{m_{2}-1} p_{\eta}(k)+p_{\eta}\left(\left(n-4 s_{2}-1\right) / 4\right) \sum_{k=0}^{s_{2}} p_{\eta}(k)=0, \\
& \sum_{k=0}^{n-1} p_{\eta}(k)=\sum_{\ell=0}^{n / m_{2}-1} \sum_{k=0}^{m_{2}-1} p_{\eta}\left(\ell m_{2}+k\right)=\sum_{\ell=0}^{n / m_{2}-1} p_{\eta}\left(\ell m_{2}\right) \sum_{k=0}^{m_{2}-1} p_{\eta}(k)=0,
\end{aligned}
$$

which means that $\Phi_{m_{2}}(q)$ divides the sums $\sum_{k=0}^{(n-t) / d} p_{q}(k)$ and $\sum_{k=0}^{n-1} p_{q}(k)$. Similarly, the two sums are also divisible by $\Phi_{m_{2}}(-q)$, By the relation

$$
\prod_{m_{2} \mid n, m_{2}>1}\left(\Phi_{m_{2}}(q) \Phi_{m_{2}}(-q)\right)=[n]_{q^{2}}
$$


we obtain

$$
\sum_{k=0}^{M}[8 k+1]_{q^{2}}[8 k+1]^{2} \frac{\left(q^{2} ; q^{8}\right)_{k}^{2}\left(a q^{2} ; q^{8}\right)_{k}\left(q^{2} / a ; q^{8}\right)_{k}}{\left(q^{8} ; q^{8}\right)_{k}^{2}\left(a q^{8} ; q^{8}\right)_{k}\left(q^{8} / a ; q^{8}\right)_{k}} q^{-4 k} \equiv 0 \quad\left(\bmod [n]_{q^{2}}\right),
$$

where $M=(n-1) / 4$ or $n-1$. Since $[n]_{q^{2}}, a-q^{2 n}$ and $1-a q^{2 n}$ are pairwise relatively prime polynomials, we complete the proof of the theorem.

Proof of Theorem 2. As same as the proof of Theorem 1, letting $a \rightarrow 1$ in (3.5), we can see that the denominator of (3.5) is relatively prime to $\Phi_{n}\left(q^{2}\right)$. On the other hand, $\Phi_{n}\left(q^{2}\right)^{2}$ is the factor of the limit of $\left(1-a q^{2 n}\right)\left(a-q^{2 n}\right)$ as $a \rightarrow 1$. Thus, we get that (1.7) holds modulo $\Phi_{n}\left(q^{2}\right)^{3}$. Meanwhile, letting $a \rightarrow 1$ in (3.8), we see that (1.7) is also true modulo $[n]_{q^{2}}$. Hence, the $q$-supercongruence (1.7) holds true. Furthermore, for $(n-1) / 4<k \leq n-1$, $\left(q^{2} ; q^{8}\right)_{k}^{4} /\left(q^{8} ; q^{8}\right)_{k}^{4} \equiv 0\left(\bmod \Phi_{n}\left(q^{2}\right)^{4}\right)$, we get $(1.8)$.

\section{Generalizations of Theorems 1 and 2}

In this section, we first give a generalization of Theorem 1 as follows.

Theorem 5. Let $n>1, d \geq 2, t$ be integers with $\operatorname{gcd}(t, d)=1$ and $n \equiv t(\bmod d)$ such that $n+d-n d \leq t \leq n$. We have

$$
\begin{aligned}
& \sum_{k=0}^{(n-t) / d}[2 d k+t] \frac{\left(q^{t} ; q^{d}\right)_{k}^{4}}{\left(q^{d} ; q^{d}\right)_{k}^{4}} q^{(d-2 t) k} \equiv \frac{\left(q^{2 t} ; q^{d}\right)_{(n-t) / d}}{\left(q^{d} ; q^{d}\right)_{(n-t) / d}}[n] q^{t(t-n) / d} \quad\left(\bmod [n] \Phi_{n}(q)^{2}\right) ; \\
& \sum_{k=0}^{n-1}[2 d k+t] \frac{\left(q^{t} ; q^{d}\right)_{k}^{4}}{\left(q^{d} ; q^{d}\right)_{k}^{4} q^{(d-2 t) k}} \equiv \frac{\left(q^{2 t} ; q^{d}\right)_{(n-t) / d}}{\left(q^{d} ; q^{d}\right)_{(n-t) / d}}[n] q^{t(t-n) / d} \quad\left(\bmod [n] \Phi_{n}(q)^{2}\right) .
\end{aligned}
$$

It is obvious that Theorem 1 is just the special case with $d=4$ and $t=1$ in Theorem 5. Letting $d=2$ and $t=1$ in (4.1), we immediately get

$$
\sum_{k=0}^{(n-1) / 2}[4 k+1] \frac{\left(q ; q^{2}\right)_{k}^{4}}{\left(q^{2} ; q^{2}\right)_{k}^{4}} \equiv[n] q^{(1-n) / 2} \quad\left(\bmod [n] \Phi_{n}(q)^{2}\right),
$$

which is a $q$-analogue of Van Hamme's (C.2) and has been proved by Guo and Wang [12].

Proof. As same as the proof of Theorem 1, we shall first establish the following parametric generalization of (4.1):

$$
\begin{aligned}
& \sum_{k=0}^{(n-t) / d}[2 d k+t] \frac{\left(q^{t} ; q^{d}\right)_{k}^{2}\left(a q^{t} ; q^{d}\right)_{k}\left(q^{t} / a ; q^{d}\right)_{k}}{\left(q^{d} ; q^{d}\right)_{k}^{2}\left(a q^{d} ; q^{d}\right)_{k}\left(q^{d} / a ; q^{d}\right)_{k}} q^{(d-2 t) k} \\
& \equiv \frac{\left(q^{2 t} ; q^{d}\right)_{(n-t) / d}}{\left(q^{d} ; q^{d}\right)_{(n-t) / d}}[n] q^{t(t-n) / d} \quad\left(\bmod [n]\left(1-a q^{n}\right)\left(a-q^{n}\right)\right) .
\end{aligned}
$$


At first, the $q$-congruence (4.4) modulo $\left(1-a q^{n}\right)$ and $\left(a-q^{n}\right)$ follows from the summation

$$
\sum_{k=0}^{(n-t) / d}[2 d k+t] \frac{\left(q^{t} ; q^{d}\right)_{k}^{2}\left(q^{t+n} ; q^{d}\right)_{k}\left(q^{t-n} ; q^{d}\right)_{k}}{\left(q^{d} ; q^{d}\right)_{k}^{2}\left(q^{d+n} ; q^{d}\right)_{k}\left(q^{d-n} ; q^{d}\right)_{k}} q^{(d-2 t) k}=\frac{\left(q^{2 t} ; q^{d}\right)_{(n-t) / d}}{\left(q^{d} ; q^{d}\right)_{(n-t) / d}}[n] q^{t(t-n) / d}
$$

which is the specialization $q \mapsto q^{d}, a=d=q^{t}, b=q^{t-n}$ and $c=q^{t+n}$ in Rogers' nonterminating ${ }_{6} \phi_{5}$ summation (2.1). On the other hand, let $c_{q}(k)$ denotes the $k$-th term on the left-hand side of (4.4). Similarly to the proof of Theorem 3, we can further show that

$$
\begin{gathered}
\sum_{k=0}^{\frac{n-t}{d}} c_{\zeta}(k)=\frac{1}{[t]_{\zeta}} \sum_{l=0}^{\frac{n-d s_{1}-t}{d m_{1}}-1} c_{\zeta}\left(l m_{1}\right) \sum_{k=0}^{m_{1}-1} c_{\zeta}(k)+\frac{1}{[t]_{\zeta}} c_{\zeta}\left(\left(n-d s_{1}-t\right) / d\right) \sum_{k=0}^{s_{1}} c_{\zeta}(k)=0 \\
\sum_{k=0}^{n-1} c_{\zeta}(k)=\frac{1}{[t]_{\zeta}} \sum_{l=0}^{n / m_{1}-1} c_{\zeta}\left(l m_{1}\right) \sum_{k=0}^{m_{1}-1} c_{\zeta}(k)=0
\end{gathered}
$$

where $\zeta \neq 1$ is a root of $\Phi_{m_{1}}(q)$ with $m_{1} \mid n$, integer $s_{1}$ satisfies $0 \leq s_{1} \leq m_{1}-1$ and $d s_{1} \equiv-t\left(\bmod m_{1}\right)$. Then the truth of (4.4) modulo $[n]$ can be proved as same as the proof of (2.10). Thus we prove that (4.4) module $[n]\left(1-a q^{n}\right)\left(a-q^{n}\right)$ is true. The $q$-supercongruences (4.1) and (4.2) then follow by letting $a \rightarrow 1$ in (4.4) and the fact that $\left(q^{t} ; q^{d}\right)_{k}^{4} /\left(q^{d} ; q^{d}\right)_{k}^{4} \equiv 0\left(\bmod \Phi_{n}(q)^{4}\right)$ for $(n-t) / d<k \leq n-1$. This completes the proof.

We also have the following generalization of Theorem 2 .

Theorem 6. Let $n>1, d \geq 2, t$ be integers with $\operatorname{gcd}(t, d)=1$ and $n \equiv t(\bmod d)$ such that $n+d-n d \leq t \leq n$. Then, modulo $[n]_{q^{2}} \Phi_{n}\left(q^{2}\right)^{2}$,

$$
\begin{gathered}
\sum_{k=0}^{(n-t) / d}[2 d k+t]_{q^{2}}[2 d k+t]^{2} \frac{\left(q^{2 t} ; q^{2 d}\right)_{k}^{4}}{\left(q^{2 d} ; q^{2 d}\right)_{k}^{4}} q^{-4 t k} \equiv \frac{-2[t]^{2}[n]_{q^{2}}\left(q^{4 t} ; q^{2 d}\right)_{(n-t) / d}}{\left(1+q^{2 t}\right)\left(q^{2 d} ; q^{2 d}\right)_{(n-t) / d}} q^{t-2 t(n-t) / d} \\
\sum_{k=0}^{n-1}[2 d k+t]_{q^{2}}[2 d k+t]^{2} \frac{\left(q^{2 t} ; q^{2 d}\right)_{k}^{4}}{\left(q^{2 d} ; q^{2 d}\right)_{k}^{4}} q^{-4 t k} \equiv \frac{-2[t]^{2}[n]_{q^{2}}\left(q^{4 t} ; q^{2 d}\right)_{(n-t) / d}}{\left(1+q^{2 t}\right)\left(q^{2 d} ; q^{2 d}\right)_{(n-t) / d}} q^{t-2 t(n-t) / d}
\end{gathered}
$$

Obviously, the $d=4$ and $t=1$ case of this theorem reduces to Theorem 2 . Furthermore, letting $d=2$ and $t=1$, we get

$$
\sum_{k=0}^{(n-1) / 2}[4 k+1]_{q^{2}}[4 k+1]^{2} \frac{\left(q^{2} ; q^{4}\right)_{k}^{4}}{\left(q^{4} ; q^{4}\right)_{k}^{4}} q^{-4 k} \equiv-[n]_{q^{2}} \frac{2 q^{2-n}}{1+q^{2}} \quad\left(\bmod [n]_{q^{2}} \Phi_{n}\left(q^{2}\right)^{2}\right),
$$

which is a $q$-analogue of (C.2) supercongruence of Van Hamme and was already obtained by Guo [6]. 
Proof. Letting $q \mapsto q^{2 d}, a=d=q^{2 t}, b=c=q^{2 d+t}, e=q^{2 t+2 n}$ and $f=q^{2 t-2 n}$ in Watson's ${ }_{8} \phi_{7}$ transformation (3.1), we can prove that, modulo $\left(a-q^{2 n}\right)$ and $\left(1-a q^{2 n}\right)$,

$$
\begin{aligned}
& \sum_{k=0}^{(n-t) / d}[2 d k+t]_{q^{2}}[2 d k+t]^{2} \frac{\left(q^{2 t} ; q^{2 d}\right)_{k}^{2}\left(a q^{2 t} ; q^{2 d}\right)_{k}\left(q^{2 t} / a ; q^{2 d}\right)_{k}}{\left(q^{2 d} ; q^{2 d}\right)_{k}^{2}\left(a q^{2 d} ; q^{2 d}\right)_{k}\left(q^{2 d} / a ; q^{2 d}\right)_{k}} q^{-4 t k} \\
& \equiv[t]^{2}[n]_{q^{2}} \frac{\left(q^{4 t} ; q^{2 d}\right)_{(n-t) / d}}{\left(q^{2 d} ; q^{2 d}\right)_{(n-t) / d}} q^{-2 t(n-t) / d}\left(1-\frac{\left(1-a q^{2 t}\right)\left(1-q^{2 t} / a\right)}{\left(1-q^{t}\right)^{2}\left(1+q^{2 t}\right)}\right) .
\end{aligned}
$$

In the same manner as the proof of Theorem 3, we can show that

$$
\lim _{q \rightarrow \eta} \sum_{k=0}^{(n-t) / d} p_{q}(k)=\lim _{q \rightarrow \eta} \sum_{k=0}^{n-1} p_{q}(k)=0
$$

where $p_{q}(k)$ is the $k$-th term on the left-hand side of (4.10) and $\eta \neq \pm 1$ is a root of $\Phi_{m_{3}}\left(q^{2}\right)$ with $m_{3} \mid n$ and $m_{3} \geq 1$. This proves that (4.10) is true modulo $[n]_{q^{2}}\left(a-q^{2 n}\right)\left(1-a q^{2 n}\right)$. The rest of the proof is similar to that of Theorem 2 and is omitted here.

\section{A Conjecture about Swisher's (G.3)}

In the last part of Swisher's [20] paper, he conjectured a series of general congruences about Van Hamme's first 12 supercongruences, which are deemed to Dwork-type congruences, such as (G.3), for $p \equiv 1(\bmod 4)$,

$$
\sum_{k=0}^{\left(p^{r}-1\right) / 4}(8 k+1) \frac{\left(\frac{1}{4}\right)_{k}^{4}}{k !^{4}} \equiv-(-1)^{\frac{p^{2}-1}{8}} p \Gamma_{p}\left(\frac{1}{2}\right) \Gamma_{p}\left(\frac{1}{4}\right)^{2} \sum_{k=0}^{\left(p^{r-1}-1\right) / 4}(8 k+1) \frac{\left(\frac{1}{4}\right)_{k}^{4}}{k !^{4}} \quad\left(\bmod p^{4 r}\right) .
$$

Note that $(-1)^{\frac{p^{2}-1}{8}}=(-1)^{\frac{p-1}{4}}$ and $\Gamma_{p}\left(\frac{1}{4}\right) \Gamma_{p}\left(\frac{3}{4}\right)=-(-1)^{\frac{p-1}{4}}$ for $p \equiv 1(\bmod 4)$, the right-side hand of (5.1) can be written as

$$
p \frac{\Gamma_{p}\left(\frac{1}{2}\right) \Gamma_{p}\left(\frac{1}{4}\right)}{\Gamma_{p}\left(\frac{3}{4}\right)} \sum_{k=0}^{\left(p^{r-1}-1\right) / 2}(8 k+1) \frac{\left(\frac{1}{4}\right)_{k}^{4}}{k !^{4}} .
$$

Not long ago, Guo [8] and Zudilin [14] proved a number of Dwork-type supercongruences, including (B.3) and some special cases of (C.3), (E.3) and (F.3) in [20], by constructing suitable $q$-analogues. We now propose the partial $q$-analogues of (G.3). It should be pointed out that the machinery in [8,14] does not work for these q-congruences.

Conjecture 1. Let $r>1, n>1$ be integers with $n \equiv 1(\bmod 4)$. Then, modulo $\left[n^{r}\right] \prod_{j=1}^{r} \Phi_{n^{j}}(q)^{2}$, we have

$$
\sum_{k=0}^{\left(n^{r}-1\right) / 4}[8 k+1] \frac{\left(q ; q^{4}\right)_{k}^{4}}{\left(q^{4} ; q^{4}\right)_{k}^{4}} q^{2 k} \equiv \frac{\left(q^{2} ; q^{4}\right)_{\left(n^{r}-1\right) / 4}}{\left(q^{4} ; q^{4}\right)_{\left(n^{r}-1\right) / 4}} \frac{\left(q^{4 n} ; q^{4 n}\right)_{\left(n^{r-1}-1\right) / 4}}{\left(q^{2 n} ; q^{4 n}\right)_{\left(n^{r-1}-1\right) / 4}}[n] q^{(1-n) / 4}
$$




$$
\begin{aligned}
& \times \sum_{k=0}^{\left(n^{r-1}-1\right) / 4}[8 k+1]_{q^{n}} \frac{\left(q^{n} ; q^{4 n}\right)_{k}^{4}}{\left(q^{4 n} ; q^{4 n}\right)_{k}^{4}} q^{2 n k}, \\
\sum_{k=0}^{n^{r}-1}[8 k+1] \frac{\left(q ; q^{4}\right)_{k}^{4}}{\left(q^{4} ; q^{4}\right)_{k}^{4}} q^{2 k} \equiv & \frac{\left(q^{2} ; q^{4}\right)_{\left(n^{r}-1\right) / 4}}{\left(q^{4} ; q^{4}\right)_{\left(n^{r}-1\right) / 4}} \frac{\left(q^{4 n} ; q^{4 n}\right)_{\left(n^{r-1}-1\right) / 4}}{\left.\left(q^{2 n} ; q^{4 n}\right)_{\left(n^{r-1}-1\right) / 4}\right] q^{(1-n) / 4}} \\
& \times \sum_{k=0}^{n^{r-1}-1}[8 k+1]_{q^{n}} \frac{\left(q^{n} ; q^{4 n}\right)_{k}^{4}}{\left(q^{4 n} ; q^{4 n}\right)_{k}^{4}} q^{2 n k} .
\end{aligned}
$$

Letting $n=p$ and $q \rightarrow 1$ in (5.2), we immediately get

$$
\sum_{k=0}^{\left(p^{r}-1\right) / 4}(8 k+1) \frac{\left(\frac{1}{4}\right)_{k}^{4}}{k !^{4}} \equiv \frac{\left(\frac{1}{2}\right)_{\left(p^{r}-1\right) / 4}(1)_{\left(p^{r-1}-1\right) / 4}}{(1)_{\left(p^{r}-1\right) / 4}\left(\frac{1}{2}\right)_{\left(p^{r-1}-1\right) / 4}} p \sum_{k=0}^{\left(p^{r-1}-1\right) / 4}(8 k+1) \frac{\left(\frac{1}{4}\right)_{k}^{4}}{k !^{4}} \quad\left(\bmod 3^{r}\right) .
$$

In order to prove that (5.2) is a direct $q$-analogue of (G.3) modulo $p^{3 r}$, we only need to verify that

$$
\frac{\left(\frac{1}{2}\right)_{\left(p^{r}-1\right) / 4}(1)_{\left(p^{r-1}-1\right) / 4}}{(1)_{\left(p^{r}-1\right) / 4}\left(\frac{1}{2}\right)_{\left(p^{r-1}-1\right) / 4}} \equiv \frac{\Gamma_{p}\left(\frac{1}{2}\right) \Gamma_{p}\left(\frac{1}{4}\right)}{\Gamma_{p}\left(\frac{3}{4}\right)} \quad\left(\bmod p^{2 r}\right) .
$$

It is obvious that (5.3) is an equivalent form of (5.2).

\section{REFERENCES}

[1] B.C. Berndt and R.A. Rankin, Ramanujan, Letters and Commentary, History of Mathematics 9, Amer. Math. Soc., Providence, RI; London Math. Soc., London, 1995.

[2] G. Gasper and M. Rahman, Basic hypergeometric series, second edition, Encyclopedia of Mathematics and Its Applications 96, Cambridge University Press, Cambridge, 2004.

[3] V.J.W. Guo, A q-analogue of the (L.2) supercongruence of Van Hamme, J. Math. Anal. Appl. 466 (1) (2018), 749-761.

[4] V.J.W. Guo, A q-analogue of the (J.2) supercongruence of Van Hamme, J. Math. Anal. Appl. 466 (1) (2018), 776-788.

[5] V.J.W. Guo, A q-analogue of the (I.2) supercongruence of Van Hamme, Int. J. Number Theory 15 (1) (2019), 29-36.

[6] V.J.W. Guo, Common q-analogues of some different supercongruences, Results Math. 74 (2019), Art. 131.

[7] V.J.W. Guo, q-Analogues of the (E.2) and (F.2) supercongruences of Van Hamme, Ramanujan J. 49 (3) (2019), 531-544.

[8] V.J.W. Guo, q-Analogues of Dwork-type supercongruences, J. Math. Anal. Appl. 487 (2020), Art. 124022.

[9] V.J.W. Guo, A $q$-analogue of the (A.2) supercongruence of Van Hamme for primes $p \equiv 1(\bmod 4)$, Rev. R. Acad. Cienc. Exactas Fís. Nat. Ser. A Mat. RACSAM, 114 (2020), Art. 123.

[10] V.J.W. Guo, Proof of a generalization of the (B.2) supercongruence of Van Hamme through a $q$ microscope, Adv. Appl. Math. 116 (2020), Art. 102016.

[11] V.J.W. Guo and M.J. Schlosser, Some new $q$-congruences for truncated basic hypergeometric series: even powers. Results Math. 75 (2020), Art. 1.

[12] V.J.W. Guo, S.-D Wang, Some congruences involving fourth powers of central $q$-binomial coefficients, Proc. Roy. Soc. Edinburgh Sect. A 150 (2020), 1127-1138. 
[13] V.J.W. Guo and W. Zudilin, A q-microscope for supercongruences, Adv. Math. 346 (2019), 329-358.

[14] V.J.W. Guo and W. Zudilin, Dwork-type supercongruences through a creative $q$-microscope, preprint, arXiv:2001.02311.

[15] G.H. Hardy, A chapter from Ramanujan's note-book, Proc. Cambridge Philos. Soc. 21 (2) (1923), 492-503.

[16] J.-C. Liu and F. Petrov, Congruences on sums of $q$-binomial coefficients, Adv. Appl. Math. 116 (2020), Art. 102003.

[17] L. Long and R.Ramakrishna, Some supercongruences occurring in truncated hypergeometric series, Adv. Math. 290 (2016), 773-808.

[18] Y. Morita, A p-adic supercongruence of the $\Gamma$ function, J. Fac. Sci. Univ. Tokyo 22 (1975), 255-266.

[19] H.-X. Ni and H. Pan, On a conjectured q-congruence of Guo and Zeng, Int. J. Number Theory 14 (6) (2018), 1699-1707.

[20] H. Swisher, On the supercongruence conjectures of Van Hamme, Res. Math. Sci. 2 (2015), Art. 18.

[21] R. Tauraso, Some q-analogs of congruences for central binomial sums, Colloq. Math. 133 (2013), 133-143.

[22] L. Van Hamme, Some conjectures concerning partial sums of generalized hypergeometric series, in: p-Adic Func btional Analysis (Nijmegen, 1996), Lecture Notes in Pure and Appl. Math. 192, Dekker, New York (1997), 223-236.

[23] X. Wang and M. Yue, Some $q$-supercongruences from Watson's ${ }_{8} \phi_{7}$ transformation formula, Results Math. 75 (2020), Art. 71.

[24] X. Wang and M. Yue, A $q$-analogue of the (A.2) supercongruence of Van Hamme for any prime $p \equiv 3$ (mod 4), Int. J. Number Theory, in press; https://doi.org/10.1142/S1793042120500694.

[25] W. Zudilin, Ramanujan-type supercongruences, J. Number Theory 129 (8) (2009), 1848-1857.

[26] W. Zudilin, Congruences for q-binomial coefficients, Ann. Combin. 23 (2019), 1123-1135.

Department of Mathematics, Shanghai University, Shanghai 200444, P. R. China

E-mail address: lydshdx@163.com (Y. Liu), xiaoxiawang@shu.edu.cn (X. Wang) 